\title{
EL LENGUAJE COMO SENTIDO Y SIGNIFICACIÓN EN LA FILOSOFÍA DE GOTTLOB FREGE Y XAVIER ZUBIRI
}

\section{LANGUAGE AS SENSE AND MEANING IN GOTTLOB FREGE AND XAVIER ZUBIRI'S PHILOSOPHY}

\author{
Luis Arturo Martínez Vásquez \\ Universidad de Costa Rica \\ luis.martinezvasquez@ucr.ac.cr
}

Recibido: 1 de octubre / Aprobado: 30 de octubre / Publicado: 10 de noviembre, 2020

\begin{abstract}
Resumen
El presente estudio pretende exponer las nociones de signo, sentido, referencia y representación en la propuesta filosófica de Friedrich Ludwig Gottlob Frege (1848-1925), así como las de expresión, señal, signo y significación en la filosofía madura de Xavier Zubiri Apalategui (1898-1983), con la pretensión de identificar relaciones y diferencias, tanto en los puntos de partida de sus planteamientos sobre lenguaje, así como en los conceptos que articulan sus propuestas. De esta manera se concluye que Zubiri aporta tanto aspectos generales como precisiones terminológicas a la filosofía del lenguaje en la actualidad.
\end{abstract}

Palabras clave:

Gottlob Frege, Xavier Zubiri, sentido, significado, signo.

\begin{abstract}
This study aims to expose the notions of sign, meaning, reference and representation in the philosophical proposal of Friedrich Ludwig Gottlob Frege (1848-1925), as well as those of expression, signal, sign and meaning in the mature philosophy of Xavier Zubiri Apalategui (1898-1983), with the aim of identifying relationships and differences, both in the starting points of his approaches to language, as well as in the concepts that articulate his proposals. In this way, it is concluded that Zubiri contributes both general aspects and terminological details to the philosophy of language today.
\end{abstract}

\section{Keywords:}

Gottlob Frege, Xavier Zubiri, sense, meaning, sign. 


\section{INTRODUCCIÓN}

La filosofía del lenguaje se ha planteado desde sus orígenes como una búsqueda incesante de los elementos constitutivos del lenguaje. Y para lograr este cometido, ha sido imperante aproximarse a lo que el lenguaje significa, lo cual requiere una serie de elementos interpretativos que enriquecen este análisis.

A partir de lo anterior, se llevará a cabo una aproximación a dos autores que han sido relevantes -cada uno de una manera específica y por lo tanto con pocos elementos en común- en lo que compete a los conceptos fundamentales del lenguaje; a saber, Gottlog Frege y Xavier Zubiri.

Para Frege la lógica tiene como centro de su estudio lo verdadero, de forma que logre reconocer las leyes que constituyen la verdad. En este sentido, las leyes podrían interpretarse como prescripciones que se deben observar, o bien como aquello que naturalmente se establece para ser observado como verdadero; es decir, como leyes del pensamiento. La lógica se desarrollará a partir de esta segunda acepción, excluyendo de esta aquellas leyes que comportan un carácter psicológico.

Por la contraparte, el tema del lenguaje en la filosofía de Zubiri no ha adquirido mayor relevancia en sus estudiosos, al igual que la relación que se pueda encontrar entre el filósofo vasco y los filósofos del lenguaje, a pesar de que en algunas de sus primeras obras de madurez el lenguaje adquiere características muy particulares, las cuales pueden conectarse directamente con aquellos autores y temas que trabajó durante los muchos años de elaboración de su prolífica obra. Así el filósofo vasco también buscará criterios de verdad a partir del lenguaje con características que merecen la pena ser analizadas y puestas a discusión.

Por lo tanto, el presente trabajo pretende explorar los principales conceptos con los que ambos autores llevan a cabo su caracterización del lenguaje, de tal forma que sea posible acercar en alguna medida aquellas filosofías que tradicionalmente han sido caracterizadas como asintóticas.

\section{FREGE Y LA LÓGICA MODERNA}

Gottlob Frege ha sido considerado el fundador de la lógica moderna, tanto por sus contribuciones acerca de los conceptos matemáticos y lógicos, como por su influencia en autores de tanta relevancia para la filosofía 
contemporánea como Ludwg Wittgenstein, Bertrand Rusell e inclusive Edmund Husserl.

El interés radical de su pensamiento estaba en función de explicar que el lenguaje posee una estructura interna constituida por el sentido y la referencia; elementos que dan razón del significado de cada palabra. De esta manera, se aparta de las corrientes de pensamiento que desde Descartes estaban centradas en dilucidaciones metafísicas y epistemológicas, para iniciar una reflexión acerca del lenguaje en su relación con la verdad, y así: “(...) abordar cómo funcionan nuestros conceptos en la misma operación lógica-lingüística de la predicación lo que arrojará algo de luz sobre dicha verdad" (Gonzalo y Attala 2018, 152).

La lógica buscará, entonces, encontrar las leyes de lo verdadero de forma que, haciendo de lado las subjetividades del conocimiento humano, sea el lenguaje el que nos permita acceder a la verdad; en este caso no por la vía de los procesos mentales, sino de los procesos lingüísticos del ser humano.

\section{Igualdad y aprioricidad en Frege}

Además de la búsqueda de certeza de lo verdadero en el ámbito de lenguaje, el concepto de igualdad, según Frege, es el que propicia estas reflexiones. Si $a=a$ estamos hablando de una identidad, ya que se entiende que $a$ es lo mismo que $a$, lo que a su vez equivaldría a un apriori kantiano de carácter analítico. Este tipo de igualdad no produce ningún conflicto en el nivel de elaboración semántica dada la infranqueable identidad o igualdad de los mismos, en las cuales no surge ninguna duda que suponga discrepancia, de ahí que $a=a$ siempre haga palpable este principio en su realización.

Sin embargo, existe otro enunciado que hace referencia a la igualdad, pero de diferente valor cognoscitivo: $a=b$. En este sentido, con este argumento no puede siempre verificarse su aprioricidad, pero se abren grandes posibilidades de interpretación de la igualdad, ya que si $a=b$ es cierto que no habría mayor distinción entre éste y $a=a$ en el ámbito del lenguaje.

Pero, ¿qué quiere decir $\mathrm{a}=\mathrm{b}$ ? Frege (1984) responderá afirmando: “(...) es que los signos o nombres ' $a$ ' y ' $b$ ' se refieren a lo mismo y por lo tanto en la igualdad se trataría precisamente de estos signos; se afirmaría una relación entre ellos" (26). 
En el caso de la primera igualdad $(a=a)$, el axioma se traduciría por el ejemplo: una piedra es igual a una piedra; mientras que la segunda igualdad $(a=b)$ posee una profundidad mucho mayor y un nivel de comprensión que a su vez supera el semántico; por ejemplo: fuego es igual a lumbre. En el ejemplo anterior es evidente la precisión en la noción fregeana de igualdad en tanto relación de una cosa consigo misma, pero la simple aseveración de un ' $a$ ' no igualable sólo con ' $a$ ' salta a un nivel de complejidad mayor.

Así, en un primer momento se habla de una identidad y una relación de los signos o nombres ' $a$ ' y ' $b$ ', pero no sólo su relación sino en su designación; es decir la relación se establece cuando estos signos $(a, b)$ designan algo, de forma que ambos signos hacen referencia a la misma cosa designada; de lo contrario, los argumentos carecerían de valor absolutamente, ya que al ser ' $a$ ' y ' $b$ ' sólo signos, la discusión se engarza siempre con su modo de designación.

De esta manera lo designado como objeto será la forma en la que se distingan los axiomas de igualdad $(a=a, a=b)$. Además, sólo se sabrá la veracidad de un argumento axiomático en lo designado de cada una de los signos que le componen, ya que resulta evidente que los signos empleados en la elaboración de una operación de este tipo $(a, b, c, d, x)$ sólo serían simples trazos escogidos al azar que por sí mismos no dicen nada específico.

Sin embargo, esta discusión superficial de los trazos no ha sido la que ha fundamentado la filosofía del lenguaje ni las operaciones matemáticas, sino aquello que se dice con el trazo escogido. Aunque el punto central sea el tema del lenguaje, en el ámbito específico de su análisis siempre será necesario desvelar la significación de lo expresado de la manera más clara posible.

\section{EI sentido (sinn) y la referencia (bedeutung)}

Para el autor, el sentido del signo es en aquello en lo que se encuentra el modo de darse el signo. Así, el sentido de un nombre propio ${ }^{1}$ será comprendido fácilmente por quien conozca el lenguaje. Al mismo tiempo llamará referencia a lo designado del signo; es aquello que no lograremos abarcar nunca completamente respecto a un sentido específico. Por ejemplo, en la frase: 'el búho de minerva', el sentido puede expresarse en un animal específico nocturno y

1 El tema del nombre propio tendrá tanto en Zubiri como en Frege consideraciones particulares que no serán objeto de análisis en esta investigación. Sin embargo es menester indicar que nombre propio será para Frege la referencia de un objeto determinado, no un concepto ni una relación. 
volador con un sonido particular, mientras que su referencia expresaría una cantidad infinita de entidades que posean estas características.

La conexión entre estos tres elementos (signo, sentido, referencia) que componen el lenguaje será la siguiente: "al signo le corresponde un determinado sentido y a éste, a su vez, una determinada referencia, mientras que a una referencia (a un objeto), no le corresponde solamente un signo. El mismo sentido puede expresarse en diferentes lenguas, e incluso en la misma, de diversas maneras"(Frege 1984, 54). Con esta afirmación, se concluye la multiplicidad de posibilidades que se encuentran en la referencia y su univocidad respecto al signo y al mismo tiempo la no consecución de uno sobre otro; ya que hay frases con un sentido, pero sin posible referencia.

Por ejemplo, la afirmación 'el caballo alado más grande' realiza las posibilidades de tener un sentido particular, tal como el de la conjunción de un animal con una característica de otro y el tamaño que éste pueda tener: Así, no es difícil encontrar un sentido para esta afirmación en el plano intelectivo, pero en el ámbito de la referencia, las posibilidades se disparan a un nivel tal que a su vez se convierte en el problema de hallarla, ya que siempre podremos imaginarnos caballos alados más grandes, por tanto la referencia última no se encontrará.

Además, en el caso de hablar de las palabras, debe apuntarse que se puede hablar de la referencia de las palabras, pero también de las palabras mismas o de su sentido. Por ejemplo, cuando se habla de 'agua', puede ocurrir que se hable del agua de los ríos costarricense (una referencia dentro de muchas), del agua como un elemento vital de la naturaleza en su sentido más general (sentido único) o de la palabra 'agua' como un conjunto de grafemas.

Lo mismo sucedería con la expresión ' $z$ ', en lo cual debe distinguirse su sentido, su referencia y la palabra en el nivel más simple. Los tres elementos componen formas muy diversas de comprender lo que se está hablando.

Así, la distinción fregeana entre sentido y referencia aporta una sutil y profunda diferencia entre los elementos que componen el análisis del lenguaje, ya que en la multiplicidad de los planos que se logran distinguir dentro del nivel semántico, se demuestra la diferenciación de lo que pertenece al signo (grafemas: $a, b, x$ ) que no tienen valor por sí mismas; lo que incumbe al sentido de ese signo (lo que unívocamente significa este signo) como expresión objetiva del lenguaje y la referencia (multiplicidad de formas en 
que se hace evidente este sentido a partir de las experiencias de quien las recibe) como forma específica en la que este sentido puede hacerse evidente en la realidad.

\section{La representación}

Si el signo (a) da razón a un sentido (caballo), que a su vez tiene múltiples referencias (muchas posibilidades de concebir este sentido de caballo; por ejemplo, un corcel blanco en alguna hacienda ecuestre de la provincia costarricense de Guanacaste), la representación (imagen subjetiva e interna "formada a partir de recuerdos de impresiones sensibles que he tenido, y de actividades que he practicado, tanto internas como externas" (Frege 1984, 56). El caballo en que aprendí a cabalgar en la infancia) varía constantemente de acuerdo a quién lo conciba y las experiencias que ha tenido el que lo concibe.

La representación añade, entonces, una variable importante en el desarrollo del lenguaje: debe conocerse quién la realiza y en qué momento, ya que cada persona realiza su propia representación. Sin embargo, pareciera haber una línea muy delgada de separación entre lo que corresponde a la representación y lo que corresponde a la referencia. Si bien es cierto que Frege carga las tintas en el tema de la subjetividad para la representación, pareciera imposible anular la subjetividad de la referencia, ya que al ser múltiple, siempre pasa por el componente humano y sus múltiples posibilidades de interpretación.

Así, de frente a la búsqueda natural del ser humano por la realidad se integra la búsqueda de la representación: “También el hombre no filosófico se ve obligado a reconocer un mundo interior diferente del mundo exterior, un mundo de impresiones sensibles, de creaciones de su imaginación, de sensaciones, de sentimientos y estados de ánimo, un mundo de inclinaciones, deseos y decisiones. Para usar una expresión breve, resumiré todo esto -exceptuando las decisiones- con la palabra 'representación'"(Frege 1996, 34). De ahí que el autor proponga algunas características de estas representaciones para distinguirlas de los objetos.

En primer lugar, estas representaciones no se pueden ver ni tocar, ni oler, ni gustar, ni oír. Se trata de impresiones que tenemos, pero que no podemos aprehender con los sentidos. Nunca tenemos aprehensión sensorial de un color, sólo de un objeto con ese color. 
Además, se tienen, como contenido de la conciencia. Tienen como momento constitutivo el sujeto que la experimenta. Sólo el sujeto que las tiene las puede determinar, ya que sólo sabremos de qué verde se habla si el sujeto lo expresa. Por ejemplo, habría grandes diferencias en la aprehensión de los colores por parte de un daltónico.

En tercer lugar, necesitan de un portador, mientras que los objetos son independientes. Y, por último, sólo poseen un portador por cada representación.

A manera de ejemplo, la radical diferencia entre representación y objeto representado lo evidencian los sentidos, ya que éstos captarán el objeto (una cuerda color rojo), pero la representación del color rojo o de una cuerda en su generalidad sólo podrán ser captadas como objetos. El color verde, una cuerda general, un caballo indeterminado son sólo representaciones realizadas por un sujeto particular y actualmente no tenemos las posibilidades de saber si todas las representaciones son iguales entre las personas; es más, sus expresiones indican lo contrario.

\section{El pensamiento}

El pensamiento, entendido como el contenido común y objetivo del pensar, contenido a su vez en un enunciado, no podría ser la referencia del enunciado, ya que la multiplicidad de posibilidades presentadas por la referencia haría caer a la persona en ambigüedades. Así, este pensamiento será el sentido del enunciado. Por ejemplo, el enunciado: 'el estagirita' será entendido en su sentido, ya que esta referencia arroja muchas posibilidades en su expresión, ya que se podría hablar de cualquier habitante singular de la ciudad de Estagira, sin embargo, su sentido lo arroja directamente a quien ha recibido este título. Al mismo tiempo, la referencia sería fuente de equívocos, ya que tanto 'el autor de la ética nicomáquea' como 'el estagirita' podrían ser, en función de la referencia, personas distintas.

Así, el pensamiento será "aquello respecto de lo cual se plantea la cuestión de la verdad” (Frege 1996, 26); además, aunque no todo el sentido de una oración sea pensamiento, en cierta forma lo es, ya que cada oración expresa un pensamiento.

En este mismo sentido, se podrían encontrar enunciados sin referencia o en los que haya partes del enunciado sin referencia, pero cuando en 
alguna parte del enunciado falta la referencia, se pierde su valor, ya que lo que interesa no es el pensamiento sino su valor veritativo ${ }^{2}$.

Ahora bien, toda propiedad que le pertenezca a un objeto se une a la propiedad de un pensamiento: la de la verdad, ya que será lo mismo decir en una oración: 'el día está soleado’ que 'es verdad que el día está soleado'; así, el sentido será igual sin haberle agregado nada a este pensamiento, que corresponde a una oración afirmativa. En este mismo sentido, se deberán distinguir tres niveles que componen a esta oración afirmativa: el pensar, el juzgar y el afirmar. Estas se encuentran relacionadas pero no indistinguibles dentro de la elaboración de la oración.

En la expresión: 'las mañanas soleadas reflejan la alegría de la primavera' se logran distinguir sus componentes: el pensar (mañanas soleadas, primavera), el juzgar (reflejar alegría primaveral) y el afirmar (es verdadero que las mañanas soleadas expresan la alegría de la primavera), lo que permite suponer al mismo tiempo que el contenido de una oración como la presentada, generalmente sobrepasa el pensamiento reflejado en ella, y en otro momento podría también no ser suficiente para expresar el pensamiento.

En este mismo sentido será necesario preguntarse si el pensamiento es una representación, sin embargo, para el autor los pensamientos no son ni objetos del mundo exterior (dada la subjetividad con la que se abordan) ni representaciones, ya que de esto dependerá el argumento de verdad con el que se desarrolle la idea. Por ejemplo, sería factible afirmar que tengo una representación de mí mismo, pero yo no soy esa representación.

\section{ZUBIRI Y LA FUNCIÓN SOCIAL DEL LENGUAJE}

Xavier Zubiri por su parte, ha desarrollado un vasto proyecto filosófico, encausado en la búsqueda de la pregunta fundamental de la filosofía, que en último término logre responder por lo constitutivo de esta disciplina, tal como a lo largo de la historia de la filosofía se ha planteado con resultados a juicio del autor muy diversos.

Así, desde sus primeros escritos se evidencia una preocupación por

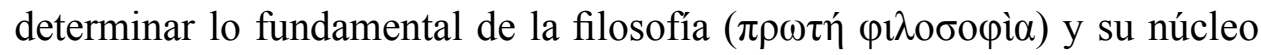

2 Para Frege, el valor veritativo será la circunstancia de un enunciado en dos posibilidades: que sea verdadero o falso, y quien emita juicios sobre los enunciados deberá hacer referencia a estos valores veritativos. Esto sucede porque en todo juicio ya se ha pasado del nivel de los pensamientos al de las referencias y sólo en el nivel de la referencia encontraremos el valor veritativo de un enunciado, ya que el en nivel del sentido, cualquier aseveración es simplemente un enunciado. 
problematizador, de tal manera que la filosofía no se confunda con otros saberes y responda a la pregunta primigenia que da razón de su reflexión.

Y en el desarrollo de su filosofía, Zubiri, al igual que Frege, ha llevado a cabo una minuciosa revisión de los conceptos con los que ha operado la filosofía, y a su vez ha insistido en la insuficiencia de lenguaje que esta disciplina posee, así como el hecho de que las muchos de los inconvenientes para una adecuada interpretación de las temáticas filosóficas radican en el lenguaje, ya que no se ha utilizado para precisar, sino por el contrario han traído serios desvíos en la historia de la filosofía occidental. Así, desde un profundo conocimiento de las lenguas clásicas ha introducido una serie de neologismos ${ }^{3}$ con el fin de ofrecer una mayor precisión terminológica al hacer filosofía.

En este proceso de reflexión, Zubiri ha rastreado los elementos fundantes de manera particular en la filosofía alemana del siglo XX. De esa forma, ha llegado a la conclusión que ni las esencias eidéticas propuestas por la fenomenología husserliana ni la noción de Dasein como ser en el pensamiento heideggeriano dan cuenta de lo primigenio del pensamiento filosófico. A pesar de que ellos han partido de la pregunta original que da comienzo a la filosofía: ¿qué es el ser? (Tí đò 'óv;), Zubiri deja claro que si la filosofía busca identificar lo primigenio, deberá partir de la realidad, ya que esta se encuentra en un nivel constitutivo previo a la noción de ser y las determinaciones de la conciencia.

De esta manera, la filosofía primera es, stricto sensu, una metafísica, entendida como una reflexión de la totalidad, una rigurosa filosofía que dé cuenta del dato más primigenio del que se pueda dar alguna explicación. Así, considera que el ser humano está irremediablemente instalado en la realidad, en la que se van imponiendo constantemente las cosas reales mediante los sentidos, los cuales no pueden no aprehender lo que tienen frente a sí, y por lo tanto, es esta aprehensión de la realidad la que permite una aproximación a la inteligencia humana y sus modo de inteligir esta realidad.

3 Además de los importantes estudios sobre el lenguaje en Zubiri que la llevado a cabo Antonio Pintor-Ramos, es importante mencionar la profundización acerca de los principales conceptos que utiliza el filósofo vasco en sus obras por parte de Germán Marquínez Argote en sus obras: Realidad y Posibilidad. Fundamentos de Ética y Educación (1995), Historia de la palabra realidad desde sus origenes latinos hasta Zubiri (2006) e Inteligencia sentiente, en perspectiva lingüistica (2013). “(...) luchando con las palabras Zubiri conformó su propia terminología filosófica, cuando los contenidos de su pensamiento así lo requerían” (Marquínez Argote, 2013, 561). 
Así, el ser humano aprehende las cosas como reales, a partir de un proceso de inteligencia sentiente; ya que es por la aprehensión intelectiva de la realidad que el ser humano toma para sí desde los sentidos estos datos primigenios, que a su vez le permiten establecer una alteridad radical, en un proceso complejo que será distinguible por la filosofía para hacer comprender su estructura, pero que en último término se imbrican de tal forma que será imposible separarlos.

Y como el ser humano está puesto en la realidad -y esta da razón de su alteridad, convirtiendo la realidad en un proceso reduplicativamente suyo-, se puede inferir que el ser humano es un animal de realidades, en el sentido en que recibe los estímulos, pero a partir de ellos forja la realidad, a la misma manera que da cuenta de su propia existencia.

\section{EI lenguaje como parte de los estudios sobre la sociedad en Zubiri}

En una de las obras más relevantes para comprender este tema, Antonio Pintor-Ramos (1987) considera que después de la publicación de Sobre la Esencia (1985) ${ }^{4}$, Zubiri podría ser cuestionado por haber dejado descuidado un análisis del lenguaje y propiciado un rechazo al giro lingüístico (Cfr. Conill Sancho, 1985), que en último término tendría como consecuencia un uso incontrolado del lenguaje que fuera -contrario a sus pretensiones- un inconveniente para comprender su filosofía.

Sin embargo, el mismo autor deja claro que tanto los conocimientos de Zubiri sobre las lenguas clásicas y modernas, como el encuentro con los filósofos de su época le han permitido la utilización del lenguaje para desarrollar su teoría de que la filosofía primera no puede ser una filosofía del lenguaje (Cfr. Corominas Escudé, 2000, 260), al mismo tiempo que el problema de la significación del lenguaje no puede ser explicado por el lenguaje mismo. Así Zubiri trasciende el nivel del lenguaje para proponer una epistemología que dé cuenta de la realidad.

Pintor-Ramos trata de demostrar que existe una progresión en el pensamiento zubiriano al comparar el curso oral de 1953-1954 denominado

4 El texto de 1985 que aquí referenciamos ha tenido cuatro ediciones previas en la Sociedad de Estudios y Publicaciones desde 1962. 
El problema del hombre, con su obra más significativa: la trilogía sobre la inteligencia sentiente 5 .

Esta progresión estaría causada al menos por dos asuntos de alta relevancia para los propósitos de esta investigación. En primer lugar, el curso oral en mención tendría una serie de construcciones teóricas que tienden a objetivarse; hasta conformar, a tenor del pensamiento de Hegel un espíritu objetivo. Esta objetivación de las construcciones teóricas sería incompatible con el análisis de lo que se actualiza en la aprehensión (Pintor Ramos 1987, 107).

Además, como consecuencia de lo anterior, se debería separar el estudio del lenguaje del estudio de la sociedad, ya que las consideraciones biológicas y antropológicas no serían necesarias para llevar a cabo el análisis de la filosofía del lenguaje.

Sin embargo, a este respecto, Antonio González (1994) considera que:

(...) no es tan claro que el contexto social en que Zubiri sitúa el problema del lenguaje sea un mero enfoque parcial del problema, del que se podría prescindir en nombre de su última filosofía. Esta tesis presupone que los estudios de Zubiri sobre la realidad social pertenecen al ámbito de la "teoría" sociológica, biológica o antropológica, y que desbordan el análisis de lo actualizado en la aprehensión. Ahora bien, aquí puede haberse introducido una confusión entre lo que desborda el análisis de la intelección y lo que desborda el análisis de lo actualizado en la aprehensión. Si ambas cosas fueran iguales, la filosofía de Zubiri se acabaría reduciendo a la noología. No hay, sin embargo, nada que justifique esto en las últimas obras de Zubiri (200).

Desde esta otra perspectiva, para Zubiri, al ser el lenguaje una realidad actualizada en la aprehensión, tiene también posibilidades de ser actualizada sin que este análisis del lenguaje se identifique con la aprehensión como tal; coincidiendo -desde su propia interpretación- con las consideraciones fregeanas de la constitución semántica del sentido que da cuenta de la referencia para llegar a un significado.

Además, constata González que la filosofía del lenguaje se ha enfocado en los últimos años en la constitución social del lenguaje, dando un paso más allá del análisis semántico, tal como Frege lo había propuesto.

5 Es decir, sus tres textos titulados: Inteligencia y Realidad (1998), Inteligencia y Lógos (1982), e Inteligencia y Razón (1984). 


\section{El ser humano como animal expresivo ${ }^{6}$}

Dentro de la vivencia colectiva, el ser humano se ve obligado por su apertura a los demás, biológicamente a través de sus funciones motoras, al igual que cualquier ser vivo, a exteriorizar desde sus funciones estimúlicas algunos signos, o señales signitivas, las cuales, en el caso de los animales quedarán como simples señales (Conill Sancho 1985, 347), mientras que en el ser humano adquieren un carácter de expresión.

Esta expresión solamente existe en alteridad. Así, en relación de colectividad, los demás irrumpen en mi vida, al punto de que por este involucramiento mis exteriorizaciones se convierten en expresiones en tanto que formas de realidad.

De esta manera, la expresión tiene varios momentos estructurales. Se pasa de expresar lo real a poner de manifiesto mi realidad, lo que a su vez funda la comunicación. Es en este momento en el que Zubiri identifica que al quedar comunicado lo expresado, esta comunicación resulta susceptible de ser interpretada. De ahí que no se trata de una simple transmisión de información, sino que quien recibe la comunicación refiere generalmente a la realidad que manifiesta esa expresión. Sin embargo, el proceso finaliza en un último momento estructural en el que quien recibe la expresión responde mediante una inevitable segunda aprehensión.

La expresión para ser tal, deberá responder a algo real, de tal manera que resulte ser reduplicativamente mío a partir de mi dimensión intelectiva. Además, la expresión, en tanto que intenta forjar algo, resulta coexpresiva, respecto de las cosas, los demás vivientes y las demás personas. Así, la expresión “(...) es una estructura real y física de mi vinculación a los demás (...), una vinculación real de mi realidad a otra realidad y en forma de realidad. Va de lo real a lo real, y es una forma de realidad (Zubiri 2007,281). Y el hombre es expresante porque actualiza su dimensión de ser expresivo y a la vez está envuelto en la expresión misma; va expreso.

En el proceso de aprehensión primordial de la realidad no interviene el lenguaje, ya que se trata de un proceso independiente de las expresiones

6 En este apartado seguiremos el análisis llevado a cabo por Zubiri en su curso oral de 1953-1954 denominado El problema del hombre, específicamente el título "el hombre, realidad social, que fue compilado posterior a la muerte del autor por Ignacio Ellacuría en 1986. Este texto, si bien no evidencia el criterio final del filósofo sobre este tema, tal como lo refiere Pintor-Ramos en el texto ya mencionado, sí permite contrastar sus ideas con la propuesta filosófica de Frege. 
fonéticas y de las interpretaciones de que esto surjan. El lenguaje será un modo de expresión que permite explicitar el dato aprehendido en los sentidos.

Por otra parte, hablar, expresar es una habitud social, al punto de que “(...) solo se puede hablar con otro" (Zubiri 2007,293), porque el lenguaje para Zubiri es un dialegein, que permite la convivencia entre los individuos. Así, el lenguaje “(...) es, ante todo y sobre todo, no un fenómeno intencional, sino un fenómeno de estructura física. Es la física de la alteridad expresada en fonaciones" (Zubiri 2007,289), lo que significa que por sí mismo, el lenguaje no es una realidad, sino una expresión de la alteridad radical del ser humano.

\section{El signo y la significación}

En este intento por expresar, el ser humano a partir de sus posibilidades biológicas establece señales, las cuales, se convierten en signos en el momento en que intentan referenciar la realidad, convirtiéndose en una huella de lo referenciado, como "una realidad expresiva que nos remite a otra realidad en forma reducida de intento" (Zubiri 2007, 287).

Dentro de este ámbito del signo, uno de ellos resulta más formalizable que los demás: el signo fonético como sonido articulado que gracias a la vida social mediante el aprendizaje de los demás permite que cobre un carácter significativo en tanto vincula dos realidades.

Estos signos, por co-volición de quienes convergen en lo signado, se transforman en símbolos, y como tales, vinculan las dimensiones signitiva y expresiva. Por lo tanto, “(...) expresión y signo es una estructura que emerge radicalmente de las estructuras de la inteligencia sentiente que el hombre posee" (Zubiri 2007, 291).

El signo remite mi propia realidad a la realidad física de lo referido. Revela lo específico de la expresión. Y la significación revela el puro ser del signo, pasando de lo hablado (signo) a lo que se dice de lo hablado (significación). Mientras que en Frege el signo se reduce a grafemas que tienen la posibilidad de ser interpretados de una u otra forma, en Zubiri adquiere un carácter dialógico, en el sentido en que la fonación hace este rodeo por el ser de la cosa para convertirse en significación.

Así, Pintor-Ramos (1987) hace notar que Zubiri no ha clarificado adecuadamente la manera en que la realidad del lenguaje aparece en la realidad primaria de los sonidos, de la misma forma en la que cabría preguntarse 
sobre la manera en la que tanto el sonido como la significación podrían articularse a partir de del hecho específico de la fonación. Así, considera que para comprender adecuadamente estas nociones, será necesario establecer una hipótesis que logre inferir esta relación:

\begin{abstract}
“(...) tal hipótesis consiste en que la relación entre sonidos y significaciones es estrictamente homogénea a la que se da entre 'cosa-realidad' y 'cosa-sentido' o, con mayor radicalidad todavía, se trata de la transformación de una 'cosa-realidad' (el sonido) en una 'cosa-sentido' (la significación), con lo que la diferencia que se da entre sentido y significación es tan solo la que media entre el todo y una de sus partes" $(117)^{7}$.
\end{abstract}

De esta manera, se inscriben los signos fonéticos en el ámbito de la realidad, a la vez que permiten una explicitación del universo interpretativo del sentido dentro de este mismo ámbito.

Por otra parte, la significación es una modificación del signo que asume de manera significativa todas las modalidades del signo, con lo cual se acerca a la definición fregeana de significado que parte del signo y que requiere de un sentido y una referencia para explicitarse. Además, ambos insisten en la dificultad propia del análisis de esta dimensión porque se ha identificado tradicionalmente la idea con el concepto, y la idea puede referir más que el concepto. La variedad de significaciones del signo fonético o bien de sentidos daría cuenta de esta pluralidad semiótica. En palabras de Pintor-Ramos (1987), en Zubiri el sentido incluye la significación, pero esto no quiere decir que la significación se reduzca a referencia de manera directa (119).

Finalmente se debe recalcar que tanto la expresión como el signo son actos de inteligencia sentiente, por eso la exteriorización animal de sonidos se queda en el nivel de lo estimúlico, mientras que el lenguaje es " (...) el órgano por excelencia de la convivencia, porque no solo manifiesta la realidad, sino que la manifiesta de modo oblicuo a través del ser" (Zubiri 2007, 296). Y al ser manifestación del ser, el lenguaje es significación. En palabras de Zubiri (2007): "El lenguaje es el órgano fonético que significa, consigna y expresa la habitud de alteridad en forma de realidad" (299).

7 Para una profundización crítica de la relación cosa-realidad /cosa-sentido en el tema del lenguaje en Zubiri, confróntese el texto de Galán (2017): Percepción sensible y lenguaje en Heidegger, Zubiri y Lonergan, 139-142. 


\section{CONCLUSIÓN}

Las propuestas de ambos autores aquí analizadas parten de diferentes consideraciones sobre la naturaleza misma del lenguaje: por una parte, Gottlob Frege considera que el sentido y la referencia son elementos constitutivos del leguaje en el nivel semántico; mientras que Xavier Zubiri ha colocado esta temática desde una consideración eminentemente antropológica y epistemológica para que dé cuenta de la dimensión social del lenguaje. Sin embargo, algunos elementos pueden considerarse afines en el momento de profundizar en sus propuestas.

En primer lugar, ambos coinciden en que la utilización simplista del lenguaje ha acarreado errores fundamentales al pensamiento occidental, como por ejemplo la mezcla inadvertida entre el plano de lo lingüístico y el de las cosas

Además, para ambos hay una necesidad de colocar la reflexión semántica dentro del campo de la realidad, ya sea mediante la noción fregeana de igualdad en la que la cosa se relaciona consigo misma, o bien la designación del lenguaje como cosa-realidad en Zubiri.

Por otra parte, las nociones fregeanas de sentido y referencia han sentado las bases para una profundización del lenguaje en sus niveles más importantes y en sus relaciones con otras disciplinas filosóficas, de modo que, a partir de su crítica, se ha venido precisando cada vez más en la historia del pensamiento contemporáneo. Estas nociones se pueden rastrear en los textos de Zubiri, las cuales ha adquirido previamente de la filosofía alemana del siglo XX.

Es así como el filósofo vasco supera la noción aristotélica del lenguaje como sonido significativo ( $\varphi$ ov̀̀ $s \varepsilon \mu \alpha \nu \tau \imath \kappa \eta ̀)$, precisando la distinción entre signo y significación, así como la subordinación de aquel a este. Además, logra distinguir entre señal, signo y significación, ampliando de esta manera la teoría fregeana de la distinción entre sentido y referencia.

Zubiri no es un filósofo del lenguaje; su propuesta va más allá, en tanto la realidad puede ser expresada y significada mediante el lenguaje; sin embargo ha aportado a la filosofía del lenguaje desde esta dimensión social, la cual permite localizar y caracterizar la función del lenguaje y su aporte a la búsqueda de la verdad, que en último término se encuentra fuera del lenguaje. 
Si bien en Zubiri el lenguaje no está en función de una filosofía primera, sí debe ser comprendido como un instrumento en función de los procesos intelectivos. Este instrumento permite una adecuada intelección y a su vez una progresión en la vida social de la persona. De esta manera, se evidencia una correlación de subordinación e interrelación entre alteridad, expresión, signo y significación.

\section{Referencias}

Conill Sancho, J. (1985). La noología de X. Zubiri. Revista de Filosofía, 8, 345-369.

Corominas Escudé, J. (2000). Ética primera. Aportación de X. Zubiri al debate ético contemporáneo. Bilbao: Desclée De Brouwer.

Frege, G. (1984). Sobre sentido y referencia. En J. Mosterin (Ed.), Estudios sobre Semántica. Barcelona: Ediciones Ariel.

Frege, G. (1996). El Pensamiento: Una investigación lógica. En M. Valdés (Ed.), Pensamiento y Lenguaje. Problemas en la atribución de actitudes proposicionales (pp. 22-48). México: Instituto de Investigaciones Filosóficas. Universidad Nacional Autónoma de México.

Galán Vélez, F. V. (2017). Percepción sensible y lenguaje en Heidegger, Zubiri y Lonergan. En-Claves del pensamiento, XI(21), 127-156.

González Fernández, A. (1994). Un solo mundo. La relevancia de Zubiri para la teoría social. Universidad Pontificia de Comillas.

Gonzalo, A., \& Attala, D. (2018). Breve reseña sobre la obra de Frege. Tópicos. Revista de Filosofía de Santa Fe, (2), 130-153. https://doi.org/https://doi.org/10.14409/ topicos.v0i2.7301

Marquínez Argote, G. (1995). Realidad y Posibilidad. Fundamentos de Ética y Educación. Santafé de Bogotá D.C.: Cooperativa Editorial Magisterio.

Marquínez Argote, G. (2006). Historia de la palabra realidad desde sus orígenes latinos hasta Zubiri. Bogotá D.C.: Editorial El Búho LTDA.

Marquínez Argote, G. (2013). Inteligencia sentiente, en perspectiva lingüística. Cuadernos Salmantinos de Filosofia, 40, 559-577. https://doi.org/10.36576/summa.32461

Pintor Ramos, A. (1987). El lenguaje en Zubiri. Cuadernos Salmantinos de Filosofía, 14, 93-133. https://doi.org/10.36576/summa.835

Zubiri, X. (1982). Inteligencia y Logos. Madrid: Alianza Editorial.

Zubiri, X. (1984). Inteligencia y Razón. Madrid: Alianza Editorial.

Zubiri, X. (1985). Sobre la esencia. Madrid: Alianza Editorial.

Zubiri, X. (1998). Inteligencia sentiente. Volumen I inteligencia y realidad. Madrid: Alianza Editorial.

Zubiri, X. (2007). Sobre el hombre (2da reimpr; I. Ellacuría, Ed.). Madrid: Alianza Editorial. 